\title{
THE INFLUENCE OF RACE AND EMOTION ON COGNITION AND METACOGNITION OF FACIAL PICTURES ${ }^{1}$
}

\author{
Zhaolan Li, Wenwu Dai, Peiyao Cong, \& Ning Jia \\ College of education, Hebei Normal University (China)
}

\begin{abstract}
In our daily life, the ability of processing the other people's facial features (such as race, emotion, etc.) are of great significance of us to adapt to social environment and participate in social interaction. In this study, a 2 (race: own-race/ other-race) $\times 2$ (emotion: positive/ negative) within-subjects design was used to investigate how the race and emotion on face affect the processing of cognition and the processing of metacognition. There are five tasks: ease-of-learning (EOL) judgement, remembering, judgement of learning (JOL), recognition and judgement of confidence (JOC). The results revealed that :(1) EOL judgement was only affected by race, which showed that participants made higher EOL judgement for other-race faces than for own-race. (2) The processing fluency was only affected by emotion, which showed that participants spend less time for learning the faces with negative emotion. (3) JOL is not only affected by race, but also moderated by emotion. The results showed that: in the positive emotion condition, JOLs of foreign faces was significantly higher than that of native faces, whereas, in the condition of negative emotion, the difference between the two was not significant. (4) Other-race effect was found in recognition scores, and the other-race effect was moderated by emotion. The results showed that the recognition performance of native face was significantly better than that foreign face in the negative emotion condition. In the condition of positive emotion, the difference between the two was not significant. (5) The trend of confidence judgment was the same as recognition scores. The conclusions were as follows :(1) Emotion has a significant influence on face image cod, while race information has a significant influence on face image cod, and emotional information plays a moderating role; (2) The metacognitive processing of face was influenced by multiple factors such as ethnicity, emotion and cognitive processing information. In conclusion, when processing face image, there is significant separation between cognition and metacognition at different stages, under the influence of ethnicity and emotion. In addition, this study also provides a partial explanation for the difference in accuracy between prospective and retrospective metacognitive monitoring.
\end{abstract}

Keywords: Metacognition, cognition, other-race effect.

\section{Introduction}

Face recognition has always been an important research direction in social cognition. The process of face recognition is affected by many factors, such as the race, emotion and so on. As an important clue to face recognition, ethnic information on faces has always been one of the research hotspots in face recognition, and the other-race effect is one of the main research directions. Other-race effect, also known as own-race bias, is a special phenomenon in face recognition, which refers to that it is easier $\mathrm{f}$ people to recognize their own race face than that of unfamiliar faces from another race. Previous studies have shown that this phenomenon is stable in various populations and experimental paradigms (Meissner \& Brigham, 2001). According to the theory of social classification, the reason why people have the other-race effect is that individuals tend to perceive the ethnic identity of the face first, and allocate their attention resources mainly to the ethnic characteristics of the face, while ignoring the personal information (such as age, emotion, etc.) carried by the face. However, when individuals perceive their own race face, they will pay more attention to the personal features of the face and less to the ethnic features (Levin., 1996; Levin, 2000).

Emotions are also important cues for individuals to recognize faces. The influence of emotion on face memory can be explained by the theory of emotional memory trade-off (Zou, Zhou, Zhang, Zhang,

\footnotetext{
${ }^{1}$ This work was supported by Humanities and Social Science Research Project of Hebei Education Department (ZD202109)
} 
\& Luo, 2011), which holds that the emotional information of the face is the main information, while the ethnic characteristics, age and gender of the face are the secondary information. In other words, in the processing of foreign faces, more attention may be paid to the emotion of the face while ignoring the facial features unrelated to emotion (such as ethnic group, etc.). Therefore, because individuals pay attention to the emotion of the face and ignore the processing of ethnic information, the other-race effect is weakened.

In our real life, people deal with more complex situations. In face recognition, a variety of cues usually need to be processed, which may inhibit or promote each other. Some studies have found that the processing of ethnic and emotional information of faces at the same time may influence each other. But it's not clear how these two factors affect different phase of the cognitive process.

At present, the research on the influence of ethnic and emotional information on face recognition is mainly focused on the cognition, while there are a few researches on metacognition. Metacognition is the cognition of cognition, including metacognition monitoring and metacognition control. Metacognitive monitoring refers to individual cognition, retrieval and judgment of cognitive process. Metacognitive control refers to the adjustment and processing of individual cognitive process by integrating various factors (Flavell\&Wellman, 1977). The commonly used measurement indicators include ease-of-learning judgement (EOL), judgement of learning (JOL), judgement of confidence (JOC), etc. The judgment on ease refers to the judgment on the difficulty of learning materials before learning and the monitoring of the cognitive process before learning (Han, 1994). Some studies have shown that the judgment on ease depends on the processing fluency experienced by individuals (Jemstedt, Schwartz, \& J ? Nsson, 2017); Learning judgment refer to the prediction of the performance of the learned material in the subsequent recall test, and it monitors the learning process of the cognitive process (Han, 1994), which is affected by the processing fluency experienced in the learning process (Rhodes \& Castel, 2008). Confidence judgment refers to the judgment on confidence in the correctness of one's answer after the extraction of learning materials, and the monitoring of the extraction stage in the cognitive process (Han, 1994). The fluency of the extraction process will affect an individual's judgment on confidence (Robey, Dougherty, \& Buttaccio, 2017). Studies have shown that there is a separation between cognition and metacognition. Nomi, et al (2013) found that participants ' JOL scores / scored for angry face pictures was higher than that for happy face pictures, but the difference in recognition accuracy was not significant. Hourihan (2019) found through the study on positive emotions, neutral emotions and negative emotions that the participants had the highest JOL score for negative emotions and the lowest score for neutral emotions, while the results were completely opposite in terms of recognition accuracy. This may be because metacognitive judgments rely on cue that differ from cognitive phase.

In conclusion, this study aims to explore the effects of ethnic and emotional information on face cognitive processing at different cognitive phase, and whether these effects can be detected or affected by metacognitive monitoring.

\section{Method}

\subsection{Participants}

31 students from a university in Hebei Province (28 valid data, including 23 female students and 5 male students). The subjects ranged in age from 17 to 27 years old $(\mathrm{M}=22.9, \mathrm{SD}=2.69)$ and were all right-handed.

\subsection{Materials}

A total of 80 face pictures were used in the experiment, among which 40 face pictures of native nationality (including 20 positive emotion pictures and 20 negative emotion pictures with a balanced ratio of male and female) were selected from the Chinese Affective Face Picture System (Gong, Huang, \& Luo, 2011). 40 alien Faces (20 positive emotions and 20 negative emotions, with a balanced gender ratio) from Karolinska's Directed Emotional Faces gallery (E.Lundqvist, Flykt, \& Öhman, 1998). The image is $260 \times 300$ pixels in size, all black and white with hair removed. Forty of these faces were used for learning, and the remaining 40 were used as distraction in the recognition phase.

\subsection{Design and procedure}

2 (race: own-race/ other-race) $\times 2$ (emotion: positive/ negative) within-subjects design was used to investigate how the race and emotion on face affect the processing of cognition and metacognition.

The experimental procedure consists of five phases:

EOL judgement. At this phase, the screen will be randomly presented a face image, each image will be presented for $1.5 \mathrm{~s}$, after that the participants need to answer this question: How easy they are to 
remember the picture just presented? (0: very not easy - 5: very easy), there is no time limit on EOL judgment, the participants need to press the number keys 0-5 to react.

Study phase. After judging all the 40 faces, participants studied these images in a new random order on the screen. The learning time is unlimited for each image. After the subject completes the memory of the face picture, he or she can enter the JOL judgment stage by pressing the space bar.

JOL judgement. After memorizing the face, the participants are required to make JOL judgment on the face image memorized, i.e. how confident they are to recognize the picture just presented in the following memory test ( 0 : no confidence at all -- 5: very confident). There is no time limit for JOL judgment, and the subject needs to press the number key 0-5 to react.

Distraction phase. After completing the self-paced learning and JOL judgment tasks, the participants played a Super Mario game for 2 minutes on a game console.

Recall phase. In this phase, the participants need to recognize a face randomly presented on the screen. They need to answer the question: Have you ever studied this picture? (1. Yes, 2. No). There is no time limit for the recognition test, and the subject needs to press the number key 1 or 2 to react. After completing the recognition test on a face picture, the subjects had to judge their confidence in the correct answer, which was called JOC phase.

JOC judgement. Make a confidence judgment on your answer, that is, how much confidence do you have that your answer is correct? ( 0 : no confidence at all -- 5: very confident) there was no time limit, and the participants had to press the number key 0-5 to react.

\section{Result}

\subsection{EOL judgement}

Table 1. EOL judgement $(M \pm S D)$.

\begin{tabular}{ccc}
\hline & \multicolumn{2}{c}{ emotion } \\
\cline { 2 - 3 } race & positive & negative \\
\hline \multirow{2}{*}{ own-race } & $3.05 \pm 0.97$ & $3.12 \pm 0.90$ \\
other-race & $3.45 \pm 0.94$ & $3.45 \pm 0.94$ \\
\hline
\end{tabular}

The results of two-factor ANOVA with ethnicity and emotion as independent variables showed that :(1) the main effect of ethnicity was significant, $F(1,27)=7.673, P=0.01, \eta_{p}{ }^{2}=0.221$. The EOL level of native face $(M=3.057)$ was lower than that of non-native face $(M=3.421)$. (2) The main effect of emotion was not significant $F(1,27)=0.366, P=0.55$; (3) The interaction between ethnicity and emotion is not significant, $F(1,27)=0.568, P=0.458$.

\subsection{Self-paced learning time}

Table 2. Self-paced learning time ( $m s) \quad(M \pm S D)$.

\begin{tabular}{ccc}
\hline & \multicolumn{2}{c}{ emotion } \\
\cline { 2 - 3 } race & positive & negative \\
\hline \multirow{2}{*}{ own-race } & $3536 \pm 2077$ & $3330 \pm 1826$ \\
other-race & $3626 \pm 1841$ & $3302 \pm 1906$ \\
\hline
\end{tabular}

Two-factor analysis of variance (ANOV) was conducted with ethnicity and emotion as independent variables. The results showed that:(1) the main effect of emotion was significant, $F(1,27)=5.527, p=0.969, \eta_{p}{ }^{2}=0.170$. The self-set speed learning time of positive emotion $(M=3407.880)$ was significantly longer than that of negative emotion $(M=3150.089)$. (2) The main effect of population was not significant, $F(1,27)=0.002, P=0.026$; (3) The interaction was not significant $F(1,27)=0.057$, $P=0.814$. 


\subsection{JOL judgement}

Table 3. JOL judgement ( $M \pm S D)$.

\begin{tabular}{ccc}
\hline & \multicolumn{2}{c}{ emotion } \\
\cline { 2 - 3 } race & positive & negative \\
\hline \multirow{2}{*}{ own-race } & $3.08 \pm 0.88$ & $3.34 \pm 0.80$ \\
other-race & $3.39 \pm 0.96$ & $3.40 \pm 0.95$ \\
\hline
\end{tabular}

The results of two-factor analysis of variance (ANOV) using ethnicity and emotion as independent variables showed that :(1) ethnicity had a significant main effect margin, $F(1,27)=3.901$, $P=0.059, \eta_{p}{ }^{2}=0.126$, and the JOL level of alien faces $(M=3.343)$ was higher than that of native faces $(\mathrm{M}=3.134)$. (2) The main effect of emotion was not significant $F(1,27)=1.842, P=0.186$; (3) The interaction between ethnicity and emotion is significant, $F(1,27)=3.805, P=0.062, \eta_{p}{ }^{2}=0.124$. Further simple effect analysis showed that (1) The JOL level of foreign faces $(\mathrm{M}=3.318)$ was significantly higher than that of native faces $(M=2.964)$ under positive emotion condition, $F(1,27)=6.924$, $P=0.014, \eta_{p}{ }^{2}=0.010$. There was no significant difference in JOL level between native and foreign faces under negative emotion condition, $F(1,27)=0.270, P=0.608$; (2) The JOL level of negative emotion $(M=3.304)$ was significantly higher than that of positive emotion $(M=2.964)$ under the condition of native face, $F(1,27)=5.634, P=0.025, \eta_{p}{ }^{2}=0.173$; There was no significant difference in JOL level between negative and positive emotions under the condition of heterogeneous face, $F(1,27)=0.079, P=0.781$.

\subsection{Recall}

Table 4. Recall $(M \pm S D)$.

\begin{tabular}{ccc}
\hline & \multicolumn{2}{c}{ emotion } \\
\cline { 2 - 3 } race & positive & negative \\
\hline \multirow{2}{*}{ own-race } & $17.62 \pm 1.47$ & $18.04 \pm 1.52$ \\
other-race & $16.75 \pm 1.36$ & $14.88 \pm 1.83$ \\
\hline
\end{tabular}

Two-factor analysis of variance using ethnicity and emotion as independent variables showed that: (1) the main effect of ethnicity was significant, $F(1,27)=26.114, P=0.000, \quad \eta_{p}{ }^{2}=0.492$, the recognition score of native face $(M=17.183)$ was significantly higher than that of alien face $(M=15.317)$; (2) The main effect of emotion was not significant $F(1,27)=2.424, P=0.131$; (3) The interaction between ethnicity and emotion is significant, $F(1,27)=29.828, P=0.000, \eta_{p}{ }^{2}=0.525$. Further simple effect analysis showed that :(1) the recognition score of native face $(M=17.857)$ was significantly higher than that of alien face $(M=14.607)$ under negative emotion condition, $F(1,27)=43.105, P=0.000, \eta_{p}{ }^{2}=0.615$; In the positive emotion condition, there was no significant difference between the recognition scores of native faces $(M=17.036)$ and non-native faces $(M=16.321), F(1,27)=3.118, P=0.089$. (2) In the native face condition, the recognition score of negative emotion $(M=17.857)$ was higher than that of positive emotion $(M=17.036), F(1,27)=6.065, P=0.020, \eta_{p}{ }^{2}=0.183$; In the heterogeneous face condition, the recognition score of positive emotion $(M=16.321)$ was significantly higher than that of negative emotion $(M=14.607)$, $F(1,27)=18.254, P=0.000, \eta_{p}{ }^{2}=0.403$.

\subsection{JOC judgement}

Table 5 JOC judgement ( $M \pm S D)$.

\begin{tabular}{ccc}
\hline \multirow{2}{*}{ race } & \multicolumn{2}{c}{ emotion } \\
\cline { 2 - 3 } & positive & negative \\
\hline own-race & $4.13 \pm 0.60$ & $4.38 \pm 0.51$ \\
other-race & $4.14 \pm 0.49$ & $4.00 \pm 0.58$ \\
\hline
\end{tabular}


Two-factor analysis of variance (ANOVA) using ethnicity and emotion as independent variables showed that: (1) the main effect of ethnicity was significant, $F(1,27)=14.258, P=0.001, \eta_{p}{ }^{2}=0.346$. The JOC level of native face $(M=4.218)$ was significantly higher than that of non-native face $(\mathrm{M}=4.021)$. (2) The main effect of emotion was not significant $F(1,27)=0.046, P=0.831$; (3) The interaction between ethnicity and emotion is significant, $F(1,27)=40.047, P=0.000, \eta_{p}{ }^{2}=0.597$. Further simple effect analysis showed that $(1)$ the JOC level of native face $(M=4.325)$ was significantly higher than that of non-native face $(M=3.927)$ under negative emotion condition, $F(1,27)=34.475, P=0.000$; Under the positive emotion condition, there was no significant difference in JOC level between native face $(M=4.111)$ and alien face $(M=4.116) F(1,27)=.010, P=0.921$; (2) The JOC level of negative emotion $(\mathrm{M}=4.325)$ was significantly higher than that of positive emotion $(M=4.111), F(1,27)=12.957, P=0.001, \eta_{p}{ }^{2}=0.324$ in native face condition; In the heterogeneous face condition, the JOC level of positive emotion pores $(M=4.116)$ was significantly higher than that of negative emotion pores $(M=3.927), F(1,27)=6.837$, $P=0.014, \eta_{p}^{2}=0.202$.

\section{Conclusions}

The influence of race and emotional information on face images is different in cognition and metacognition, and the processing of face images shows significant separation in different cognitive and metacognitive.

\section{References}

E. Lundqvist, D., Flykt, A., \& Öhman, A. (1998). The Karolinska Directed Emotional Faces - KDEF, CD ROM from Department of Clinical Neuroscience, Psychology section, Karolinska Institutet, ISBN 91-630-7164-9.

Flavell, J. H., \& Wellman, H. M. (1977). Metamemory. In R. V. Kail, Jr \& J. W. Hagen (Eds.). Perspectives on the development of memory and cognition. New York: Halsted Press

Gong X, Huang YX, \& Luo YJ. (2011). The revision of Chinese facial expression image system. Chinese Journal of Mental Health, 25(001), 40-46.

Han, K. (1994). Theoretical framework of metamemory research. Advances in Psychological Science (1), 29-336.

Hourihan, K. L. Misleading emotions: judgments of learning overestimate recognition of negative and positive emotional images

Jemstedt, A., Schwartz, B. L., \& Jönsson, F. U. (2017). Ease-of-learning judgments are based on both processing fluency and beliefs. Memory, 1-9.

Johnson K J, Fredrickson B L. "We all look the same to me": Positive emotions eliminate the own-race bias in face recognition. Psychological Science, 2005, 16(11): 875-881

Levin DT. Classifying faces by race: The structure of face categories $[\mathrm{J}] . J$ Exp Psychol: Learn, Mem Cogn, 1996, 22: 1364-1382.

Levin DT. Race as a visual feature: Using visual search and perceptual discrimination tasks to understand face categories and thecross-race recognition deficit [J].J Exp Psychol General, 2000, 129: 559-574.

Meissner, C. A., \& Brigham, J. C. (2001). Thirty years of investigating the own-race bias in memory for faces: A meta-analytic review. Psychology, Public Policy, \& Law, 7, 3-35.

Nomi, Jason, S., Rhodes, Matthew, G., Cleary, \& Anne, M. (2013). Emotional facial expressions differentially influence predictions and performance for face recognition. Cognition and Emotion.

Robey, A. M., Dougherty, M. R., \& Buttaccio, D. R. (2017). Making retrospective confidence judgments improves learners' ability to decide what not to study. Psychol, 956797617718800.

Zou, J. L., Zhou, R. L., Zhang, H., Zhang, X. C., \& Luo, X. Y. (2011). Emotional memory trade-offs effect: memory for complicated emotional stimuli. Advances in Psychological Science. 